\title{
Umwelttoxikologie
}

\section{Arsen in Grundwasser und Reis - Ursachen und Konsequenzen}

\author{
HANNA JOSS ${ }^{1}$, E. MARIE MUEHE ${ }^{2}$, ANDREAS KAPPLER ${ }^{1}$ \\ ${ }^{1}$ GEOMIKROBIOLOGIE, ZENTRUM FÜR ANGEWANDTE GEOWISSENSCHAFTEN, \\ UNIVERSITÄT TÜBINGEN \\ 2 PFLANZEN-BIOGEOCHEMIE, DEPARTMENT UMWELTMIKROBIOLOGIE, \\ HELMHOLTZ ZENTRUM FÜR UMWELTFORSCHUNG, LEIPZIG
}

\section{The toxic metalloid arsenic (As) is present in the environment often associated with iron(III) oxide minerals. Arsenic can be mobilized into groundwater by iron(III)-reducing, and thus, mineral-dissolving bacteria. We investigate in situ natural organic matter and methane as electron donors fueling microbial iron(III) reduction, the removal of As by iron oxides in drinking water filters, and the effect of climate change on redox processes in the rice rhizosphere and on uptake of As into rice.}

DOI: $10.1007 / \mathrm{s} 12268-020-1452-9$

(C) Die Autoren 2020

Das toxische Halbmetall Arsen kommt auf natürliche Weise weltweit in Sedimenten und Böden vor. Eine Kombination verschiedener geochemischer und mikrobieller Prozesse führt zur Freisetzung von Arsen, sodass Arsen über das Grundwasser in Trinkwasser und Reis und damit in die Nahrungskette gelangt. Weltweit wird so die Gesundheit von mehr als 100 Millionen Menschen gefährdet.

In der Natur ist Arsen vor allem an schwerlösliche Eisen(III)-Oxid-Minerale gebunden. Kompetitive Desorption durch Phosphat und natürliches organisches Material (NOM), $\mathrm{pH}-$ Wert-Änderungen, aber vor allem mikrobielle Reduktion von Eisen(III) und Arsen(V) führen zur Mobilisierung von Arsen. Eine zentrale Rolle spielen hier eisenreduzierende Mikroorganismen. Unter anoxischen Bedingungen verwenden sie Eisen(III) als Elektronenakzeptor zur Atmung, reduzieren es dadurch zu Eisen(II) und lösen dabei die Minerale auf, sodass vorher gebundenes Arsen in das Grundwasser freigesetzt wird [1].

Durch die Bohrung von Grundwasserbrunnen seit den 70er-Jahren in Süd- und Südostasien nehmen die Menschen Arsen über das Trinkwasser auf, wodurch es zur „weltweit größten Massenvergiftung der Menschheit“ kam [2]. Bis heute verursacht Arsen in diesen Regionen Krankheitsbeschwerden wie
Hautausschläge oder Kreislaufbeschwerden und kann bei chronischer Exposition Hautkrebs auslösen und sogar zum Tod führen.

Ziele unserer Forschung sind daher, die Mechanismen der Freisetzung von Arsen in der Umwelt besser zu verstehen, zur Entwicklung von Methoden beizutragen, um Arsen effektiv und praktikabel aus Trinkwasser zu entfernen und abzuschätzen, wie sich die Mobilisierung und die Anreicherung von Arsen in der Nahrungskette unter zukünftigen klimatischen Bedingungen entwickelt.

\section{Mikrobielle Mobilisierung von Arsen} in das Grundwasser - Rolle von organischem Material und Methan

Die Freisetzung von Arsen in das Grundwasser geschieht vor allem durch die mikrobielle Auflösung von arsenhaltigen Eisen(III)Mineralen unter sauerstofffreien Bedingungen. Eisen(III)-reduzierende Mikroorganismen benötigen für diesen Atmungsprozess einen Elektronendonor, z. B. organischen Kohlenstoff. In bisherigen Studien wurden meist kurzkettige Fettsäuren (Acetat, Laktat) und damit gut bioverfügbare Verbindungen eingesetzt. Wir haben untersucht, ob diese Mikroorganismen auch durch Oxidation von komplexeren organischen Verbindungen, wie sie vor Ort im Untergrund vorkommen, Eisen(III) reduzieren und Arsen freisetzen.
Wir haben dazu aus Bohrungen in der Nähe von Hanoi, Vietnam, Sedimentkerne gewonnen (Abb. 1) und daraus organisches Material extrahiert. Laborexperimente zeigten, dass Mikroorganismen unter Verwendung des im Sediment vorhandenen organischen Materials in gleicher Weise, und potenziell sogar mehr Arsen freisetzen können, als mit zugegebenen leicht verfügbaren Kohlenstoffquellen [3]. Im Vergleich zu Laktat/Acetat konnten wir zeigen, dass die mikrobielle Nutzung der im Sediment vorhandenen organischen Verbindungen zu einer höheren mikrobiellen Diversität führt. Daraus wurde für Laborexperimente geschlussfolgert, dass zur Untersuchung der für die Arsenfreisetzung relevanten Prozesse zukünftig natives NOM aus den Grundwasserleitern verwendet werden sollte.

Derzeit untersuchen wir erstmalig auch die Rolle von Methan als Elektronendonor für die mikrobielle Auflösung von arsenhaltigen Eisen(III)-Mineralen. Methan ist häufig in großen Mengen in arsenkontaminierten Aquiferen vorhanden. Laborexperimente haben gezeigt, dass Methan in den AquiferSedimenten von methanotrophen Eisen(III)reduzierenden Mikroorganismen oxidiert wird und so durch die Auflösung der Eisen(III)Minerale zur Arsenmobilisierung beitragen kann.

\section{Entfernung von Arsen mittels Sandfilter}

In Nordvietnam verwendet die Bevölkerung einfache Sandfilter (Abb. 2), um das Arsen $\left(\mathrm{As}^{3+}\right)$ und das im Wasser ebenfalls enthaltene gelöste Eisen $\left(\mathrm{Fe}^{2+}\right)$ und Mangan $\left(\mathrm{Mn}^{2+}\right)$ aus dem Wasser zu entfernen, und das Wasser für den Hausgebrauch nutzen zu können. Dabei wird das anoxische, kontaminierte Grundwasser in den oberen Teil des Filters geleitet, der Sand z. B. aus dem Roten Fluss enthält. Wir konnten zeigen, dass eine Kombination von chemischen und mikrobiellen Prozessen dazu führt, dass $\mathrm{Fe}^{2+}$ und $\mathrm{Mn}^{2+}$ mit Luftsauerstoff oxidiert werden [4]. Das Eisen fällt als schwerlösliches Eisen(III)-Mineral auf den Sandkörnern aus und bindet das 
Arsen, welches so zusammen mit dem $\mathrm{Fe}^{2+}$ und $\mathrm{Mn}^{2+}$ aus dem Trinkwasser entfernt wird. Derzeit untersuchen wir, woran optisch erkannt werden kann, ob der Filter effizient das Arsen entfernt. Es wurde beobachtet, dass die Oxidation des $\mathrm{Mn}^{2+}$ zur Bildung von schwarzen $\mathrm{Mn}(\mathrm{IV})$-Oxid-Mineralen $\left(\mathrm{MnO}_{2}\right)$ führt. Solange $\mathrm{Fe}^{2+}$ und gelöstes $\mathrm{As}^{3+}$ im Wasser sind, werden diese durch das $\mathrm{MnO}_{2}$ oxidiert, als Eisen(III)-Mineral und sorbiertes Arsen(V) gebunden. Dadurch werden die Mn(IV)-Minerale wieder aufgelöst. Erst wenn alles $\mathrm{Fe}^{2+}$ und $\mathrm{As}^{3+}$ oxidiert und gebunden ist, bleiben die schwarzen $\mathrm{MnO}_{2}$-Minerale bestehen. Mit Analysen der Filter in Vietnam und Säulenexperimenten im Labor untersuchen wir derzeit, ob das Vorhandensein dieser schwarzen Schicht ein Indikator dafür ist, dass der Filter effizient das Arsen aus dem Wasser entfernt. Für die Bevölkerung wäre das eine einfache visuelle Kontrollmöglichkeit, um die Notwendigkeit des Austauschs des Filtermaterials zu überprüfen.

\section{Arsen in Reis}

Arsenbelastetes Grundwasser wird in vielen Regionen auch zur Bewässerung von Reisfeldern verwendet, wo es durch Aufnahme in die Reispflanzen zur Anreicherung des Arsens im Reis kommt. Da Reis für etwa die Hälfte der Weltbevölkerung das Grundnahrungsmittel darstellt, ist es wichtig die Prozesse zu verstehen, welche die Mobilität und Aufnahme des Arsens in die Reispflanze beeinflussen (Abb. 3). Durch den Transport von Luftsauerstoff durch die Wurzeln der Reispflanze in den Boden kommt es zur Oxidation von $\mathrm{Fe}^{2+}$ und zur Ausfällung von Eisen(III)-Mineralen entlang von Reiswurzeln. Unsere Arbeiten haben gezeigt, dass Eisen(II)-oxidierende Mikroorganismen vermehrt in der Rhizosphäre vorkommen und wesentlich zur Bildung der Eisen(III)-Minerale auf den Wurzeln (Eisenplaque) beitragen $[5,6]$. An diesen Eisenmineralen kommt es zur gleichzeitigen Bindung von Arsen, was wiederum die Aufnahme des Arsens in den Reis reduziert.

Der Reisertrag wird meist durch Stickstoffdüngung gesteigert, wodurch es zu Denitrifikation und der Freisetzung von Zwischenprodukten wie Nitrit und Lachgas (Distickstoffmonoxid, $\mathrm{N}_{2} \mathrm{O}$ ), aber auch zur Oxidation von $\mathrm{Fe}^{2+}$ und Bildung von Eisen(III)-Mineralen durch nitratreduzierende eisenoxidierende Bakterien kommt. Durch die abiotische Reduktion von Nitrit durch Eisen(II) (Chemodenitrifikation) kommt es zur Bildung und

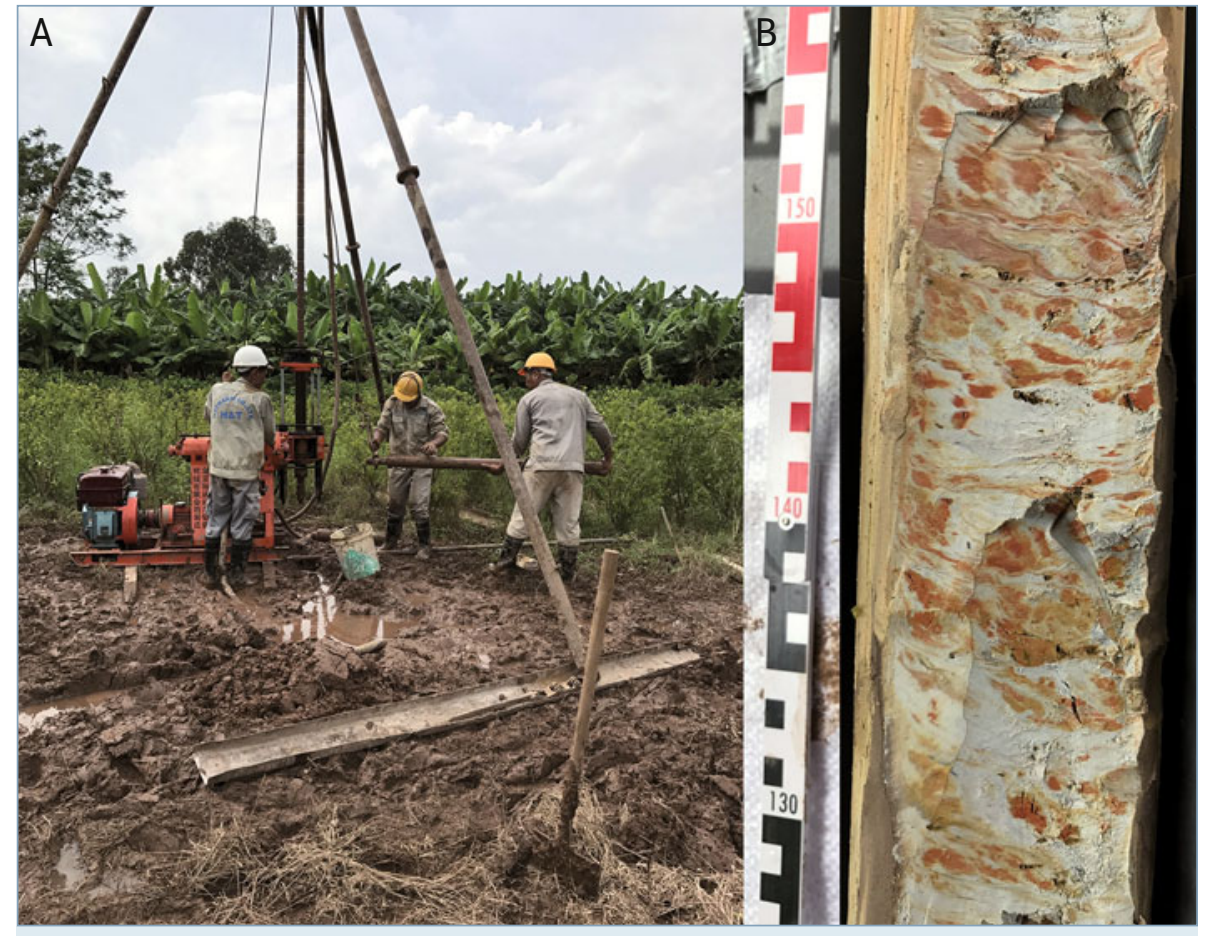

$\Delta$ Abb. 1: Arsen im Boden. A, Grundwasserbohrung nahe des Dorfs Van Phuc, südöstlich von Hanoi, Vietnam. B, Bohrkerne $(\mathrm{d}=10 \mathrm{~cm})$ wurden aus bis zu $46 \mathrm{~m}$ Tiefe entnommen. Rostrote Bereiche, welche den Bohrkern durchziehen, sind charakteristisch für arsenhaltige Eisen(III)Minerale. Fotos: Andreas Kappler.

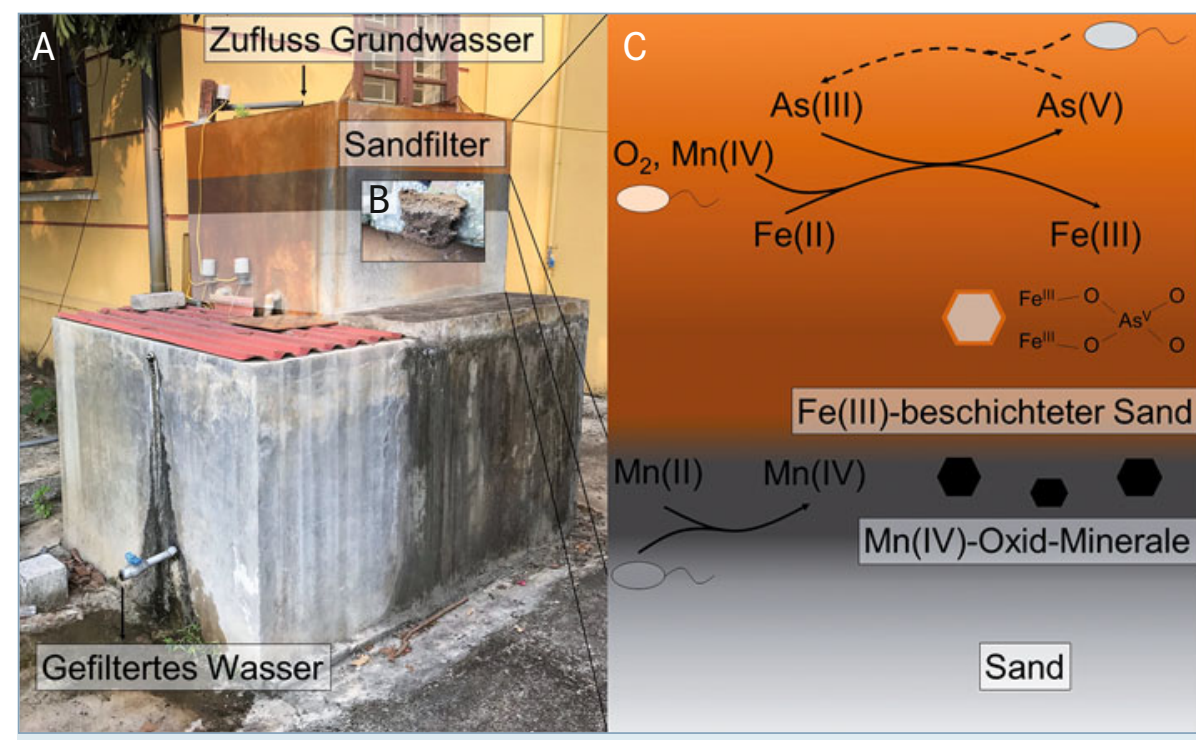

A Abb. 2: Arsen im Trinkwasser. A, Trinkwasserfilter in Vietnam mit Zufluss von arsenkontaminiertem Grundwasser in den oberen Betonkasten, der Sand aus dem Roten Fluss enthält. Das Wasser fließt durch den Sand in das untere Becken zur Speicherung des gefilterten Wassers.

B, Material aus dem Trinkwasserfilter. C, Abiotische und biotische Prozesse, die in den einzelnen Schichten des Trinkwasserfilters ablaufen. Fotos: Andreas Kappler.

Emission von $\mathrm{N}_{2} \mathrm{O}$. In einem neuen Projekt untersuchen wir in chinesischen Reisfeldböden, inwieweit die dosierte Zugabe von Stickstoffdünger gleichzeitig die Arsenimmobilisierung durch die gebildeten Eisen(III)Minerale maximiert und die Freisetzung des
Klimagases $\mathrm{N}_{2} \mathrm{O}$ minimiert. Somit soll abgeschätzt werden, mit welcher Düngungspraxis die Risiken für die Bevölkerung und das Klima so gering wie möglich sind.

Neben dem Effekt der Düngung untersuchen wir die Auswirkungen der Klimaverän- 

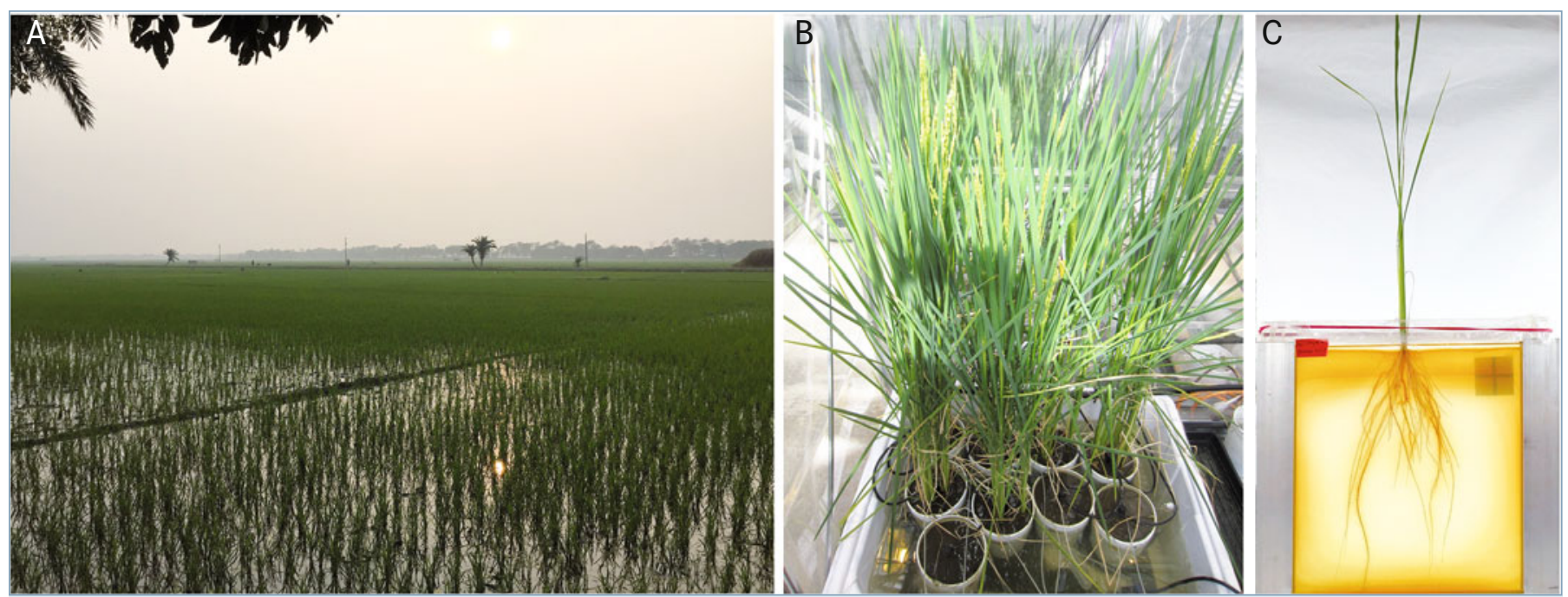

Abb. 3: Versuch zur Aufnahme von Arsen durch Reispflanzen. A, Reisfeld in Vietnam. B, Kultivierung von Reispflanzen in Gewächshäusern, um die Auswirkung von klimatischen Veränderungen zu untersuchen. C, Zeitlich aufgelöste Aufnahme von räumlichen biogeochemischen Veränderungen in der Wurzelzone in einem Rhizotron. Fotos: Andreas Kappler, E. Marie Muehe, Markus Maisch.

derungen auf die Arsenaufnahme in den Reis. So konnten wir zeigen, dass unter einem sich ändernden Klima, wie es derzeit für 2100 vorhergesagt wird, die Aktivität von Eisen(III)- und Arsen(V)-reduzierenden Mikroorganismen gefördert wird und zu vermehrter Arsenfreisetzung in der Rhizosphäre der Reispflanzen führen würde [7]. Dies kann wiederum dazu führen, dass sich die Reisqualität durch den Anstieg des Arsengehalts im Reiskorn verschlechtern und der Ertrag von Reis deutlich sinken wird. Deshalb gilt es Lösungen zu finden, welche die gekoppelten Belastungen durch Arsen im Boden und das veränderte Klima mildern.

\section{Danksagung}

Wir bedanken uns bei allen ehemaligen und derzeitigen Mitarbeitern, die zu den Forschungsarbeiten maßgeblich beigetragen haben, insbesondere bei Markus Maisch, Martyna Glodowska, Anh Van Le, Monique Patzner und dem AdvectAs Team. Besonderer Dank gilt der Deutschen Forschungsgemeinschaft, dem Marie-Curie-Programm der EU und der Universität Tübingen für die finanzielle Unterstützung unserer Arbeit.

\section{Literatur}

[1] Muehe EM, Kappler A (2014) Arsenic mobility and toxicity in South and South-east Asia - a review on biogeochemistry, health and socio-economic effects, remediation and risk predictions. Environ Chem 11:483-495

[2] Smith AH, Lingas EO, Rahman M (2000) Contamination of drinking-water by arsenic in Bangladesh: a public health emergency. Bull World Health Organ 78:1093-1103 [3] Glodowska M, Stopelli E, Schneider M et al. (2020) Role of in situ natural organic matter in mobilizing As during microbial reduction of FeIII-mineral-bearing aquifer sediments from Hanoi (Vietnam). Environ Sci Technol 54:4149-4159 [4] Nitzsche KS, Lan VM, Trang PTK et al. (2015) Arsenic removal from drinking water by a household sand filter in Vietnam - effect of filter usage practices on arsenic removal efficiency and microbiological water quality. Sci Total Environ 502:526-536

[5] Maisch M, Lueder U, Kappler A, Schmidt C (2019) Iron lung: how rice roots induce iron redox changes in the rhizosphere and create niches for microaerophilic Fe(II)-oxidizing bacteria. Environ Sci Technol Lett 6:600-605

[6] Maisch M, Lueder U, Kappler A, Schmidt C (2020) From plant to paddy - how rice root iron plaque can affect the paddy field iron cycling. Soil Syst 4:28

[7] Muehe EM, Wang T, Kerl CF et al. (2019) Rice production threatened by coupled stresses of climate and soil arsenic. Nat Commun 10:4985

Funding Open Access funding enabled and organized by Projekt DEAL. Open Access Dieser Artikel wird unter der Creative Commons Namensnennung 4.0 International Lizenz veröffentlicht, welche die Nutzung, Vervielfältigun Bearbeitung, Verbreitung und Wiedergabe in jeglichem Medium und Form
erlaubt, sofern Sie den/die ursprünglichen Autor(en) und die Quelle erlaubt, sofern Sie den/die ursprünglichen Autor(en) und die Quelle
ordnungsgemäß nennen, einen Link zur Creative Commons Lizenz beifügen und angeben, ob Änderungen vorgenommen wurden. Die in diesem Artikel enthaltenen Bilder und sonstiges Drittmaterial unterliegen ebenfalls der genannten Creative Commons Lizenz, sofern sich aus der Abbildungslegende nichts anderes ergibt. Sofern das betreffende Material nicht unter der genannten Creative Commons Lizenz steht und die betreffende Handlung nicht nach gesetzlichen Vorschriften erlaubt ist, ist für die oben aufgefüh Weiterverwendungen des Materials die Einwilligung des jeweiligen Rechteinhabers einzuholen. Weitere Details zur Lizenz entnehmen Sie bitte der
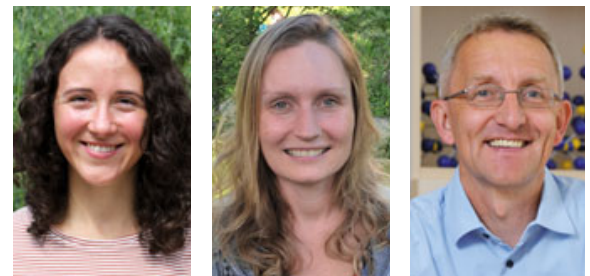

Hanna Joss, E. Marie Muehe und Andreas Kappler (v.I.n.r.)

Korrespondenzadresse:

Prof. Dr. Andreas Kappler

Geomikrobiologie

Zentrum für Angewandte Geowissenschaften

Universität Tübingen

Schnarrenbergstraße 94-96

D-72076 Tübingen

andreas.kappler@uni-tuebingen.de

https://uni-tuebingen.de/fakultaeten/

mathematisch-naturwissenschaftliche-fakultaet/ fachbereiche/geowissenschaften/arbeitsgruppen/ angewandte-geowissenschaften/angewandtegeowissenschaften-zag/geomikrobiologie/ workgroup/ 